ISSN 1981-416X

Licenciado sob uma Licença Creative Commons

\title{
Lycée nouveau départ: conduite et dispositif paradoxaux: une approche socio- linguistique du discours d'un responsable
}

\author{
Second Chance High School: conduct \\ and paradoxical device: a socio-linguistic \\ approach to a responsible's speech
}

\author{
François Gouraud, Bertrand Bergier
}

Université Catholique de l’Ouest, Angers, France

\section{Résumé}

Cet article étudie le récit des pratiques d'un enseignant responsable d'un lycée Nouveau Départ accueillant des élèves qui, après avoir décroché, retournent aux études L'approche paradoxale systémique (Barel, 1989) permet de souligner une des caractéristiques de ces structures atypiques: celle d'être hors des normes scolaires habituelles tout en préparant à une épreuve de certification du système scolaire, ce qui implique l'acquisition d'un contenu en référence à un diplôme de l'éducation nationale et l'intériorisation des exigences impersonnelles de sa passation. Décryptant les justifications des pratiques du responsable face à l'absentéisme - principale expression de l'indiscipline dans un Lycée Nouveau 
Départ - notre étude montre que c'est en ayant la conduite paradoxale de «viser l'impossible» que, lui et son équipe, remplissent leur mission.

Mots-clés: Lycée Nouveau Départ. Justification. Responsable. Conduite paradoxale.

\section{Abstract}

This article examines the story of the practices of a teacher in charge of a Second Chance High School (SCHS) which welcomes students who are going back to school, after having dropped out. The paradoxical systemic approach (Barel, 1989) makes it possible to emphasize one of the characteristics of these atypical structures: that of being outside the usual school standards while preparing for a certification test of the school system, which implies the acquisition of content in reference to a diploma of the Départment of National Education and the internalization of impersonal requirements of its graduation. Decrypting the justifications of the SCHS manager's practices regarding absenteeism - the main expression of indiscipline in a Second Chance High School - our study shows that it is by having the paradoxical drive to "aim for the impossible» that he and his team, fulfill their mission.

Keywords: Second Chance High School. Justification. Responsible. Paradoxical conduct.

\section{Introduction}

En France, pour permettre à des jeunes qui sont sortis du système scolaire sans diplôme, de reprendre le chemin des études et d'obtenir un premier titre académique tel un baccalauréat, ont été créés des structures appelées notamment Lycée Nouvelle Chance (secteur public) ou encore Lycée Nouveau Départ (secteur privé). Si nombre de travaux portent sur les «décrocheurs»(Bloch; Gerde, 2004; Blaya, 2010; Bernard, 2017), plus rares sont ceux s'intéressant aux «raccrocheurs» (Melin, 2012; 
Zaffran; Vollet, 2018), qui plus est aux enseignants-responsables de ces lycées atypiques. Dans cet article, nous étudions le sens que confère un enseignant-responsable d'un Lycée Nouveau Départ (LND) à ses pratiques professionnelles. Cette recherche exploratoire vise à documenter la question de recherche suivante: Etre enseignant et responsable d'un lycée Nouveau Départ, quelle parole pour donner sens à son action, au regard des exigences académiques et professionnelles intériorisées?

\section{Quête de sens: systèmes de justification, paradoxes et stratégies}

\section{Quel montage composite et quelles stratégies langagières pour décrire des pratiques d'un Lycée Nouveau Départ (LND)?}

Comment les discours, produits lors d'un entretien de recherche, par un enseignant-responsable d'un LND, sont-ils ou non révélateurs d'un «montage composite» (Derouet, 1988; 1989) où entrent en tension les modèles civique, domestique et industriel en éducation? Les mots utilisés pour décrire ce que cet enseignant-responsable perçoit, pense et fait ne sont pas sans lien avec la façon de se représenter les différentes situations de sa pratique.

\section{Posture de justification}

Lors de l'entretien semi-directif - défini comme un espace social de justification en référence à des modèles (Derouet, 1988, p. 68-70) quatre thèmes ont été abordés: «Les missions de l'enseignant vis-à-vis du public accueilli dans le Lycée Nouveau Départ» «la transgression du règlement», «une production scolaire en deçà des attentes de l'enseignant», «La préparation du jeune à l'ordre impersonnel de l'examen du bac». Nous traiterons, dans le cadre de cet article, le thème de la transgression qui se manifeste au LND à travers l'absentéisme des décrocheurs-raccrocheurs. 


\section{Trois références}

Pour analyser le discours produit par l'enseignant-responsable d'un LND, nous prenons appui sur le trépied suivant: la déclinaison des systèmes de justification construit par Jean Louis Derouet (1988; 1989), les idées de paradoxe et système d'Yves Barel (1982; 1989) et l'interprétation des discours en référence à l'analyse sémiotique décrite par Greimas et Courtès (1993).

\section{Des systèmes de justification des logiques d'action du métier d'enseignant}

Pour Jean Louis Derouet, «Chacun (des enseignants) doit trouver son identité en composant des éléments générés dans des logiques contradictoires et qui ne demandent qu'à se dénoncer mutuellement... Ce travail est compliqué par le fait que les enseignants doivent maintenant travailler à l'intersection de plusieurs systèmes de justification dont les principes, les références, les instruments, sont assez sensiblement différents» (1988, p. 69). Derouet identifie trois systèmes de justification, en lien avec les modèles de compétences sociales plus généraux, construits par Luc Boltanski et Laurent Thévenot (1991), à savoir: «le modèle civique, le modèle domestique et le modèle industriel». Voici comment il les décrit.

Le modèle civique

Le modèle civique fonde la définition administrative du fonctionnaire... L'importance des personnes s'y mesure à leur capacité à se déprendre des intérêts particuliers, locaux et personnels, et à incarner l'intérêt général... Le pire défaut, dans un tel système, est de faire acception des personnes et de céder aux pressions de l'affection ou des interconnaissances locales. Cet effort pour se déprendre du réseau des influences locales est une nécessité logique du modèle...Mais l'exigence d'exterritorialité de l'école reste fondamentale... Le même souci de rupture préside à la définition des savoirs: les savoirs de l'école sont des savoirs généraux 
d'autant plus respectable qu'ils sont plus formels et plus abstraits, et qu'ils n'ont rien à voir avec les pratiques et les objets du quotidien...Le but de l'école est de libérer les hommes - ...- du joug des préjugés familiaux et des servitudes locales... (DEROUET, 1988, p. 63-64).

Le modèle domestique

L'établissement et la classe sont conçus sur un modèle familial, et l'enseignant doit se comporter en père - ou de mère- de ses élèves. Dans ce cas, la justice ne repose pas sur l'application impersonnelle de règles générales, mais au contraire sur la connaissance très personnelle des individus, et éventuellement sur la faveur...la pédagogie peut consister à compenser par des relations affectives privilégiées un handicap qui éloigne un élève de l'école... (DEROUET, 1988, p. 64-65).

Le modèle industriel

...C'est dans ce contexte qu'il faut comprendre la popularité du terme de professionnalisation. Il correspond bien entendu à une montée générale d'un modèle industriel de l'éducation...Le but des enseignants, dans la professionnalisation de leur métier, est sans doute l'efficacité dans des situations qui deviennent de plus en plus problématique; c'est aussi s'armer face à un débat social qui les envahit. (DEROUET, 1988, p. 67).

Cependant, si Jean-Louis Derouet différencie les systèmes de justification utilisés par les enseignants, il ne montre pas comment ceux-ci procèdent pour tenir ensemble des références de justification potentiellement contradictoires à partir de situations concrètes.

\section{L'approche paradoxale systémique, le paradigme de référence}

De nombreux écrits en sciences humaines font référence à la figure du paradoxe (Bateson, 2008; Barus-Michel, 2013; Watzlawick, 2014). Celle-ci est définie et mobilisée différemment selon les objets de recherche, la discipline et les ancrages épistémologiques des auteurs. 
Nous retenons le positionnement d'Yves Barel. Sa thèse est la suivante nous disent Amiot, Billard et Brams (1993):

Sa pensée pour l'essentiel peut se résumer dans l'idée que les contradictions dont le réel est fait sont insupportables et indépassables, mais que, néanmoins la vie impose de les gérer. Gérer l'impossible: cette idée n'est pas simple, mais double ou paradoxale (AMIOT, BILLARD, BRAMS, 1993, p. 13).

Il utilise les figures du «paradoxe» et du «système» pour tenter d'avoir une intelligence de la façon dont les acteurs sociaux construisent un sens aux contradictions qui déterminent leur situation sociale.

Le paradigme, légué par Yves Barel, nous aide à penser les déterminants des situations sociales et les stratégies mises en œuvre pour maîtriser plus ou moins - et non contrôler ou dépasser — une situation paradoxale.

\section{Stratégies simple ou paradoxale}

Yves Barel a identifié quatre stratégies paradoxales: de compromis, d'oscillation, de triangulation et de compartimentage. La stratégie de compromis consiste à tenir ensemble, dans le même espace temps, les déterminants qui définissent la situation paradoxale. La stratégie d'oscillation tient ensemble les déterminants par un mouvement de va et vient. La stratégie de triangulation permet la co-présence des déterminants grâce à la mobilisation d'un système tiers. Enfin, la stratégie de compartimentage permet de tenir ensemble les déterminants, en déléguant à certains acteurs, dans certains espaces temps, la gestion d'un seul de ceux-ci.

Cependant, l'acteur social peut aussi ne pas assumer le paradoxe de la situation sociale dans laquelle il se trouve. Il développe alors une stratégie simple, dont les caractéristiques sont de ne retenir que l'un des déterminants, ou encore aucun. L'effet produit est alors, non d'annuler la situation paradoxale, mais de la déplacer et de déléguer la responsabilité de la maîtrise du paradoxe à d'autres acteurs sociaux. 


\section{L'indécidable et le processus d'actualisation potentialisation}

Pour Yves Barel, ce qui caractérise la situation paradoxale de la production et de la reproduction des conditions d'une adaptation d'un système social, c'est l'indécidable. C'est choisir, à un moment donné, l'un des déterminants du paradoxe, sans pour autant détruire l'autre terme. Le processus est à l'œuvre. L'acteur influe sans détruire les autres logiques qui définissent la situation sociale, tout en privilégiant une orientation. Ainsi, la construction d'un sens des pratiques en référence à trois systèmes de justification (DErouet, 1988), peut alors être pensée comme l'actualisation momentanée d'un système, sans pour autant détruire les autres systèmes de justification. L'actualisation d'un des systèmes potentialisent les autres qui peuvent, à la suite, voire aussi en même temps, être convoqués.

\section{Méthode par «incongruité»}

Yves Barel indique qu'il utilise la méthode par «incongruité» ou rapprochement paradoxal de choses, élaborée par Kennet Burke (1989, p. 48), pour mettre au jour les processus à l'œuvre, lors de la quête de sens des acteurs sociaux. En effet, pour lui, une compréhension différente peut-être dévoilée, si l'on tente de penser le rapport construit, par "un assemblage insolite ou inhabituel de mots. Un tel assemblage produit un effet dans la mesure où il «révèle des "relations entre les objets que notre vocabulaire rationnel coutumier à ignore" (Burke, 1935, p. 90). Parler d'un lion comme d'un chien..., c'est utiliser la perspective par incongruité" (Roig, 1977, p. 72). Il choisit cette perspective, plutôt que de poursuivre le travail de distinction entre les éléments qui composent un chien et un lion.

Nous retenons, de la figure du paradoxe existentiel et du système construit par celui-ci, que ce qui apparaît comme distinct peut aussi fusionner, et ce qui apparaît comme fusionnant contient des unités distinctes. C'est l'expression d'un processus d'actualisation potentialisation. Le visible cache de l'invisible. Les systèmes qui composent une situation 
paradoxale à la fois se distinguent et se contiennent. De plus, un système contient en lui-même sa propre contradiction.

Concrètement, il s'agira alors, pour nous, d'observer comment le narrateur met en scène, à l'aide des mots utilisés, la situation évoquée et la stratégie mise en œuvre pour y apporter une réponse. Nous identifierons les termes employés comme contraire par le narrateur et ceux qui expriment leur rapport. Nous repèrerons en quoi les réponses décrites permettent ou pas de tenir ensemble les termes contraires de la situation évoquée.

\section{L’analyse sémiotique, une quête d'indices}

Pour autant, Yves Barel, ne nous a pas transmis d'indications méthodologiques plus précises pour identifier, à partir des discours, les déterminants de la situation paradoxale et le type de stratégie mis en œuvre par l'acteur social.

Aussi, dans un premier temps, nous avons recours à l'analyse classique de contenu par catégorie. Ce découpage nous permet de repérer l'existence ou non des trois systèmes de justification proposés par Jean Louis Derouet (1989). Elle offre aussi l'occasion de percevoir comment s'effectue le passage de l'un à l'autre en lien avec les thèmes ou sous thèmes abordés.

Puis, nous avons eu recours à l'analyse sémiotique de Greimas et Courtès (1993) pour l'interprétation d'extraits. Cette dernière nous permet de mettre au jour d'une façon plus fine - à partir de son analyse des ressorts langagiers de la construction d'un sens à l'aide des mots — des indices. Ceux-ci nous permettent d'identifier les termes en tension, qui définissent la situation évoquée par le narrateur. Il facilite le repérage du type ou des types de stratégie que celui-ci met en œuvre pour dissocier et tenir ensemble les déterminants de la situations évoquée.

L'analyse sémiotique se décompose en trois classes: narrative, discursive et énonciative. La première est composée: du programme narratif, des actants, de la performance principale, et du carré sémiotique. La 
deuxième: le parcours figuratif et la performance principale. La dernière renvoie à l'énonciation.

C'est donc en prenant appui sur ce dispositif à la fois théorique et méthodologisue que nous proposons d'étudier la façon dont un enseignant-responsable d'un lycée Nouveau Départ (ND) justifie son action. Nous identifierons les termes qui structurent la situations décrite - en l'occurrence l'absentéisme d'un élève - et les réponses ou stratégies mises en oeuvre.

\section{La situation: système de justification, déterminants et réponses}

Interrogé sur le type d'infraction au règlement dans un lycée Nouveau Départ, le responsable mentionne l'absentéisme. Il évoque les absences répétées d'un élève ( $1^{\text {er }}$ sous-thème) mais aussi sa difficulté à se mettre au travail ( $2{ }^{\text {ème }}$ sous-thème), puis fait un retour critique sur son admission au LND ( $3^{\text {ème }}$ sous-thème).

Narration: actualisation et potentialisation des systèmes de justification.

Voici, à partir de l'analyse par catégorie que nous avons effectuée, pour chacun des sous thèmes, une illustration du mouvement imprimé au discours par le narrateur, au regard des trois logiques:

- civique (A) ou la référence au règlement de l'éducation nationale,

- familiale (B) ou l'attention à la singularité de l'élève,

- industrielle $(C)$ ou l'efficacité qui implique une compétence professionnelle.

Nous les présentons à l'aide du modèle de la hiérarchie enchevêtrée. Celle-ci suggère qu'il n'y a pas de hiérarchie entre les systèmes: celui qui est actualisé potentialise les autres et ainsi de suite. 
Pour le premier sous thème, nous avons les mots de transitions du passage d'un niveau ou système à l'autre en 2 et en 4 . Les chiffres indiquent le déroulement du discours.

\section{"L'absentéisme d'un élève»}

A: 1-c'est encore moi qui ai sa convocation au BAC. C'est-à-dire qu'aujourd'hui, il ne peut pas passer son bac.

5- je lui ai dit qu’il lui faudrait venir la chercher lui-même.

4- Là du coup. 2-Alors, évidemment

B: 3- je l'ai appelé, je l’ai relancé.

Je lui ai dit que bien sûr il pouvait venir la chercher. En réalité son camarade m'a demandé s'il pouvait prendre sa convocation.

C: 10 ...C'est représentatif de l'infraction au règlement telle qu'on peut la connaître au lycée ND.

Nous constatons un mouvement de va et vient de la logique réglementaire à la logique domestique ou familiale. Voyons maintenant un extrait du deuxième sous thème. 


\section{"Avoir énormément de mal à se mettre au travail»}

\section{A 8- honnêtement, que pour le bac}

B 1- Pour moi, comment dire, c'est un élève qui a toujours eu énormément de mal à se mettre au travail.

3-Parce qu'il a été présent, il a quand même fait un effort de présence. Alors c'est toujours un petit peu compliqué. Il fallait que je le relance quand même très, très régulièrement là-dessus. Mais il a quand même fait un effort tout au long de l'année.

5- Et c'est un élève qui, à mon avis, a du mal à se mettre au travail.

7- Et je crains

C 2- C'est la question de la mise au travail, c'est la question de s'astreindre: s'astreindre à une présence, s'astreindre à un travail.

4-Par contre, la présence ne veut pas dire forcément le travail.

6- Je crois qu'il y a quand même une vraie difficulté de ce côté-là.

9- ce soit extrêmement compliqué. Peuvent se permettre de faire cela (ne pas se mettre au travail) des élèves qui ont des vraies facilités. Certains d'entre eux peuvent se permettre de faire cela et de réussir en fin d'année. Mais à mon avis, il n'entre pas dans ce cadre-là.

Pour ce passage, le mouvement d'oscillation s'opère essentiellement entre la logique industrielle et la logique familiale. Enfin, voici le troisième sous thème consacré au retour critique sur les critères d'admission au LND. 


\section{"L'une des erreurs dans cette histoire-là est..."}

A: 3-Néanmoins, il y avait un cas de figure où on pouvait proposer une certaine souplesse en termes d'organisation.

8-C'est pour ça qu'on ne ferme jamais la porte à cette idée-là.

B: 2- Il n'a pas été déscolarisé. Souvent c'est quelque chose pour laquelle on est très attentif.

4- Ce qui a fait qu'on lui a proposé.

6- oui mais j'ai un contre exemple, pas plus tard que cette année.

C: 1- En fait, je pense que l'une des erreurs dans cette histoire-là est de l'avoir accueilli directement après une terminale classique.

5- Souvent le passage direct d'une Terminale classique à une structure un peu alternative comme la nôtre est rarement, (pas toujours) pertinent.

7- C'est difficile de dégager des règles immuables.

Nous pouvons, pour le troisième sous thème, voir le mouvement s'opérer entre les trois niveaux ou logiques de justification.

Ces trois exemples illustrent le mouvement de va et vient du discours du narrateur entre les différents systèmes de justification. Cette façon d'opérer lui permet d'actualiser une des logiques et de potentialiser les autres logiques, sans les détruire.

Nous avons retrouvé ce même procédé pour les différents thèmes évoqués dans l'interview.

L'analyse par catégorie confirme donc l'hypothèse de Jean-Louis Derouet (1989), à savoir le recours à des systèmes de justification différents par ce responsable d'un lycée Nouveau Départ pour décrire ce qu'il perçoit, pense et fait.

Quand l'absence contient la présence, la difficulté la présence sans la mise au travail ou l'absence sans la facilité, l'erreur la pertinence. 
Nous vous présentons, maintenant, le résultat d'une des catégorisations de l'analyse discursive, les composantes sémantiques, afin d'approcher la façon dont celui-ci qualifie ces trois types de situation et le type de réponse ou stratégie qu'il construit.

L'analyse discursive est l'une des trois catégories de l'analyse sémiotique. Les composantes sémantiques sont structurées par trois catégories ou niveaux: figuratif, thématique et axiologique.

\section{«...l’absentéisme d'un élève...».}

Au niveau axiologique, la situation est caractérisée comme: "représentative de l'infraction au règlement telle qu'on peut la connaître au LND”.

Le premier sous thème, au niveau thématique, est structuré par l'opposition: "avoir réussi à être présent" et "avoir disparu complètement depuis trois semaines".

Au niveau figuratif, l'élève est décrit, tout d'abord, comme ayant "un parcours scolaire qui a été marqué par beaucoup. de voyages, ...". Le cadre de son accueil est spécifié en lien avec la réglementation de l'éducation nationale en vigueur: "Il est venu chez nous pour passer les quelques matières qui lui manquent.... On est sur quelque chose qui est un petit peu à la carte. Ce que nous on peut faire et qu'une structure classique ne fera pas forcément.". L'action du responsable du lycée nouveau départ est mentionnée: "Alors, évidemment, je l'ai appelé, je l'ai relancé.... En réalité son camarade m'a demandé s'il pouvait prendre sa convocation... Là, pour le coup, je lui ai dit qu'il lui faudrait venir la chercher lui-même". Ainsi, le niveau figuratif illustre une connaissance détaillée du parcours de l'élève. Il décrit les attributions spécifiques du lycée ND et l'action du responsable du lycée. Celui-ci tente de rentrer en contact avec l'élève absent et tient à respecter les conditions règlementaires de la remise de la convocation au bac.

"...l'absentéisme d'un élève... (situation) représentative de l'infraction au règlement». 


\begin{tabular}{|c|c|c|}
\hline Contraire: absence & Et/ou & Contraire: présence \\
\hline $\begin{array}{l}\text { depuis trois semaines, } \\
\text { il a disparu }\end{array}$ & $\begin{array}{l}\text { et là, } \\
\text { Alors, évidemment, } \\
\text { "Là, pour le coup, }\end{array}$ & $\begin{array}{l}\text { "ll est venu chez nous pour passer les quelques } \\
\text { matières qui lui manquent.... } \\
\text { "l a réussi à être présent"... } \\
\text { je l'ai appelé, je l'ai relancé ...» } \\
\text { je lui ai dit qu'il lui faudrait venir la chercher } \\
\text { lui-même». }\end{array}$ \\
\hline
\end{tabular}

L'extrait du niveau thématique ci-dessus, illustre comment celui-ci est structuré par l'opposition: présence absence. C'est par un mouvement de va et vient: de l'absence à la présence, que le narrateur décrit la transgression typique du règlement dans un lycée ND: l'absence.

La situation paradoxale que tente de maîtriser le responsable de ce lycée, grâce au mouvement de son discours, est de rendre présent l'absent.

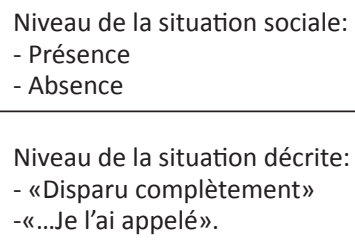

\section{«Avoir du mal à se mettre au travail»}

La deuxième sous partie est structurée, au niveau axiologique par l'idée suivante: "C'est la question de la mise au travail, c'est la question de s'astreindre: s'astreindre à une présence, s'astreindre à un travail... Peuvent se permettre de faire cela (ne pas se mettre au travail) des élèves qui ont des vraies 
facilités... il fait partie de ces élèves qui n'ont pas les facilités qui leur permettraient de pouvoir se permettre de telles absences (de travail)".

$\mathrm{Au}$ niveau thématique, par deux oppositions. La première: «la présence ne veut pas dire forcément le travail». La deuxième: «Pour moi, c'est un échec.».

Le niveau figuratif met en scène à la fois l'action de l'élève "Parce qu'il a été présent, il a quand même fait un effort de présence." et celle du responsable du lycée "Alors c'est toujours un petit peu compliqué. Il fallait que je le relance quand même très, très régulièrement là-dessus», soulignant ce qui revient à l'élève: "Mais il a quand même fait un effort tout au long de l'année". Est évoqué ensuite l'évaluation des aptitudes de l'élève: "Et c'est un élève qui, à mon avis, a du mal à se mettre au travail. Je crois qu'il y a quand même une vraie difficulté de ce côté-là" desservies par les conditions de passation du bac pour cet élève: "Il se trouve dans une situation où finalement il joue son année sur des matières dans lesquelles il n'est pas a priori au mieux". Est enfin formulé le sentiment du responsable à l'égard de cette situation singulière: "Je suis très inquiet... Pour moi c'est dommage" et son évaluation: "Pour moi, c'est un échec".

\begin{tabular}{|lll|}
\hline \multicolumn{1}{|c|}{ Contraire } & \multicolumn{1}{c|}{ Et/ou } & \multicolumn{1}{c|}{ Contraire } \\
\hline $\begin{array}{l}\text { Présence: } \\
\text { "la présence }\end{array}$ & $\begin{array}{l}\text { ne veut pas dire forcément } \\
\text { «Pour moi, } \\
\text { c'est }\end{array}$ & $\begin{array}{l}\text { Travail: } \\
\text { le travail». } \\
\text { clensemble de la situation décrite) }\end{array}$ \\
\hline
\end{tabular}

Si l'on se reporte à la description de ce passage à l'aide de la hiérarchie enchevêtrée, dans la première partie de notre développement, l'on perçoit, là aussi, que c'est grâce à l'oscillation que le narrateur dissocie et relie les termes contraires de son énoncé.

Le responsable de ce lycée tente de maîtriser, par une stratégie d'oscillation, la situation paradoxale suivante: illustrer comment l'échec contient les conditions de sa réussite. 
«Sentiment dominant: échec» ou quand l'échec contient les conditions de la réussite.

Niveau de la situation sociale: réussite

- Présence

- Travail et/ou facilité

Niveau la situation décrite: échec

- « Mais il a quand même fait un effort tout au long de l'année».

-«Pour moi, comment dire, c'est un élève qui a toujours eu énormément de mal à se mettre au travail. Je crois qu'il y a quand même une vraie difficulté de ce côté-là»»».

"L'une des erreurs»

Pour le sous-thème consacré au retour critique sur les critères d'admission, le niveau axiologique repose sur l'idée suivante: “C'est difficile de dégager des règles immuables. C'est pour ça qu'on ne ferme jamais la porte à cette idée-là.".

Le niveau thématique est structuré sur l'opposition: "l'avoir accueilli directement après une terminale classique.", "Souvent le passage direct d'une Terminale classique à une structure un peu alternative comme la nôtre est rarement, (pas toujours) pertinente".

Le niveau figuratif illustre la singularité de la situation de l'élève: "Il n'a pas été déscolarisé...", et la spécificité administrative du cadre d'un LND: (Amiot, M.; BILLARD, I.; BRAMS, L., 1993, p. 13): "Néanmoins, il y avait un cas de figure où on pouvait proposer une certaine souplesse en termes d'organisation. Ce qui a fait qu'on lui a proposé". 


\begin{tabular}{|lll|}
\hline \multicolumn{1}{|c|}{ Contraire: } & Et/ou & \multicolumn{1}{c|}{ Contraire: } \\
\hline $\begin{array}{l}\text { En fait, je pense que l'une des erreurs } \\
\text { dans cette histoire-là est de l'avoir } \\
\text { accueilli directement après une terminale } \\
\text { classique. Il n'a pas été déscolarisé }\end{array}$ & Souvent & \\
$\begin{array}{l}\text { ily avait un cas de figure où on pouvait } \\
\text { proposer une certaine souplesse en } \\
\text { termes d'organisation. Ce qui a fait qu'on } \\
\text { lui a proposé. }\end{array}$ & Néanmoins, & $\begin{array}{l}\text { c'est quelque chose pour laquelle } \\
\text { on est très attentif }\end{array}$ \\
& Souvent & $\begin{array}{l}\text { le passage direct d'une Terminale } \\
\text { classique à une structure un } \\
\text { peu alternative comme la nôtre } \\
\text { est rarement, (pas toujours) } \\
\text { pertinent. }\end{array}$ \\
$\begin{array}{l}\text { j'ai un contre exemple, pas plus tard que } \\
\text { cette année. }\end{array}$ & oui mais & $\begin{array}{l}\text { C'est difficile de dégager des } \\
\text { règles immuables. }\end{array}$ \\
$\begin{array}{l}\text { qu'on ne ferme jamais la porte à cette } \\
\text { idée-là. }\end{array}$ & C'est pour ça & \\
\hline
\end{tabular}

Ce tableau illustre l'alternance entre les contraires pour les dissocier et les tenir ensemble.

La situation paradoxale que tente de maîtriser le narrateur, par l'oscillation entre les termes contraire, c'est de faire que l'erreur qu'il décrit n’en soit pas systématiquement une.

Accueil après une terminale ou quand l'erreur n'est pas une erreur

Niveau de la situation sociale d'un Lycée Nouveau Départ

- "Le passage direct d'une Terminale classique à une structure un peu alternative comme la nôtre est rarement, (pas toujours) pertinent».

- "C'est difficile de dégager des règles immuables".

Niveau la situation décrite: I'une des erreurs

- "L'avoir accueilli directement après une terminale classique».

- "...On ne ferme jamais la porte à cette idée-là».

Ce développement illustre comment le discours produit par le responsable d'un lycée ND sur le thème de l'infraction au règlement, 
identifiée comme l'absence, tente de maîtriser, plus ou moins, trois facettes de cette situation, qui sont structurées par des pôles antagonistes, grâce à une stratégie d'oscillation qui les dissocient et relient:

- celui de la présence de celui qui est absent,

- celui d'une difficulté qui contient une présence sans travail ou facilité,

- et, enfin, celui d'une erreur qui peut, parfois, être pertinente.

L'absence, - qui est en même temps quête d'une présence, et l'erreur, —qui peut en même temps être pertinente, sont deux des aspects de la situation dont l'issue est indécidable. En effet, peut être que l'élève viendra ou pas, peut être que l'erreur pour cet élève n'en sera pas une pour un autre. Nous avons là un des signes d'une situation paradoxale, l'impossibilité de trancher entre les termes.

Voici, d'une façon synthétique les résultats de l'interprétation construite du premier thème abordé lors de l'entretien, l'indiscipline.

\begin{tabular}{|lllll|}
\hline & $\begin{array}{l}\text { Termes } \\
\text { contraires } \\
\text { à tenir } \\
\text { ensemble ou } \\
\text { déterminants } \\
\text { des situations. }\end{array}$ & $\begin{array}{l}\text { Discours et } \\
\text { systèmes de } \\
\text { justification } \\
\text { mobilisés. }\end{array}$ & $\begin{array}{l}\text { Stratégie simple } \\
\text { ou paradoxale }\end{array}$ & $\begin{array}{l}\text { Question et/ } \\
\text { ou conduite } \\
\text { paradoxale ou } \\
\text { simple. }\end{array}$ \\
\hline $\begin{array}{l}\text { 1-1 Infraction } \\
\text { au règlement } \\
\text { dans un LND: } \\
\text { «l'absentéisme } \\
\text { d'un élève... } \\
\text { représentative } \\
\text { de l'infraction } \\
\text { au règlement». }\end{array}$ & $\begin{array}{l}\text { Absence. } \\
\text { Réglementation la remise de } \\
\text { la convocation } \\
\text { au bac. }\end{array}$ & $\begin{array}{l}\text { Oscillation } \\
\text { entre la logique } \\
\text { familiale ou la } \\
\text { prise en compte } \\
\text { de la singularité } \\
\text { de chaque élève } \\
\text { et la logique } \\
\text { réglementaire ou } \\
\text { civique. }\end{array}$ & $\begin{array}{l}\text { Stratégie } \\
\text { d'oscillation } \\
\text { entre prendre } \\
\text { contact et ne } \\
\text { rien céder } \\
\text { sur l'exigence } \\
\text { règlementaire. }\end{array}$ & $\begin{array}{l}\text { Comment } \\
\text { rendre présent } \\
\text { l'absent tout } \\
\text { en remplissant } \\
\text { les impératifs } \\
\text { règlementaires? } \\
\text { Ou quand } \\
\text { l'absent se fait } \\
\text { présent. }\end{array}$ \\
\hline
\end{tabular}




\begin{tabular}{|c|c|c|c|c|}
\hline $\begin{array}{l}\text { 1-2 "Avoir } \\
\text { énormément de } \\
\text { mal à se mettre } \\
\text { au travail» }\end{array}$ & $\begin{array}{l}\text { Présence. } \\
\text { et } \\
\text { Travail } \\
\text { ou } \\
\text { Absence } \\
\text { et } \\
\text { Facilité. }\end{array}$ & $\begin{array}{l}\text { Oscillation } \\
\text { entre logique } \\
\text { de l'efficacité ou } \\
\text { industrielle et } \\
\text { logique familiale. }\end{array}$ & & $\begin{array}{l}\text { Pourquoi cet } \\
\text { élève ne réussit- } \\
\text { il pas? Ou } \\
\text { quand l'échec } \\
\text { décrit contient } \\
\text { les conditions } \\
\text { de la réussite. }\end{array}$ \\
\hline $\begin{array}{l}1-3 \text { "L'une des } \\
\text { erreurs dans } \\
\text { cette histoire-là } \\
\text { est...» }\end{array}$ & $\begin{array}{l}\text { Accueillir après } \\
\text { une terminale } \\
\text { et } \\
\text { Pertinence. }\end{array}$ & $\begin{array}{l}\text { Oscillation entre } \\
\text { les trois systèmes } \\
\text { de justifications: } \\
\text { la logique civique, } \\
\text { familiale et } \\
\text { industrielle. }\end{array}$ & $\begin{array}{l}\text { Stratégie } \\
\text { d'oscillation } \\
\text { entre erreur et } \\
\text { pertinence. }\end{array}$ & $\begin{array}{l}\text { Comment ce } \\
\text { qui est une } \\
\text { erreur pour } \\
\text { un élève en } \\
\text { n'est pas une } \\
\text { pour d'autres? } \\
\text { Ou quand une } \\
\text { erreur est et } \\
\text { n'est pas une } \\
\text { erreur. }\end{array}$ \\
\hline
\end{tabular}

Le thème de l'absence, comme expression de l'indiscipline, peut paraître surprenant. Cependant, le constat de ce responsable d'un lycée nouveau départ est concordant avec les observations de Joël Zaffran et Juliette Vollet lors de leur enquête: “D'ailleurs, nous n'avons assisté à aucune scène d'indiscipline, de chahut ou de violence dans les salles de cours ou dans l'enceinte du bâtiment. Les exclusions, quand elles interviennent, sont davantage liées à un absentéisme excessif" (2018, p. 77).

\section{Conclusion: limites et mise en perspective}

Cette analyse sociolinguistique, de type qualitative, présente l'intérêt d'être, à notre connaissance, la première interprétation d'un entretien d'un responsable d'un Lycée Nouveau Départ, à partir des références mobilisées. Elle a comme limite d'être effectuée à partir d'un seul entretien d'un responsable d'un lycée ND. En cela elle ne peut donc pas prétendre à une généralisation, seulement à une illustration. Mais que nous montre-elle? Elle nous révèle des situations et des réponses paradoxales. Ainsi, c'est en alternant entre la prise de contact et le rappel de l'exigence réglementaire de 
la remise de la convocation au bac que ce directeur tient ensemble la prise en compte de la singularité de l'élève absent et la logique réglementaire qui s'applique à tous. C'est aussi en oscillant entre erreur et pertinence qu'il laisse la porte ouverte à l'accueil après une terminale, même s'il avance que, pour le cas présenté, ce fut une erreur. Autant de pratiques paradoxales révélatrices d'une conduite paradoxale celle de "viser l'impossible". Cette logique peut avoir le sens de vouloir faire que tous les élèves accueillis puissent avoir leur chance, celle d'avoir un diplôme, pour être à égalité, dans la recherche d'un emploi (ZAFFRAN; VOLLET, 2018, p. 38).

\section{Références}

AMIOT, M.; BILLARD, I.; BRAMS, L. Système et paradoxe. Autour de la pensée d’Yves Barel. Paris: Seuil, 1993.

BAREL, Y. Les enjeux du travail social. Actions et Recherches Sociales, n. 3, p. 2340, nov. 1982.

BAREL, Y. Le paradoxe et le système. Essai sur le fantastique social. Grenoble: PUG, 1989.

BARUS-MICHEL, J. L'énergie du paradoxe. Paris: DDB, 2013.

BATESON, G. Vers une écologie de l'esprit. Tome 2. Paris: Seuil-Point, 2008.

BERGIER, B.; FRANCEQUIN, G. La revanche scolaire: Des élèves multiredoublants, Bernard, P-Y. Le décrochage scolaire. Paris: PUF, 2017.

BLAYA, C. Décrochages scolaires, l'école en difficulté. Bruxelles: De Boeck Pédagogies en développement, 2010.

BLOCH, M-C.; GERDE, B. Un autre regard sur les décrocheurs. Le CLEPT, une expérience de rescolarisation en France. Revue internationale déducation, v. 35, p. 89-97, 2004.

BOLTANSKI, L.; THÉVENOT, L. De la justification. Les Economies de la grandeur. Paris: Gallimard, 1991. 
BURKE, K. Permanence and Change: An Antomy of Purpose. 2. ed. Indianapolis/ New Yorks: Bobbs-Merrill, 1989.

DEROUET, J-L. La profession enseignante comme montage composite. Les enseignants face à un système de justification complexe. Education Permanente, $\mathrm{n}$. 96, p. 61-71, 1988.

DEROUET, J-L. Les établissements scolaires dans leur environnement: une entreprise composite. Actes du colloque de Tours, v. 25-26, p. 19-43, avr. 1989.

GREIMAS, A. J.; COURTÈS, J. Sémiotique. Dictionnaire raisonné de la théorie du langage. Paris: Hachette Livre, 1993.

MELIN, V. Le raccrochage scolaire une épreuve biographique dans le processus de subjectivation d'anciens décrocheurs en situation de raccrochage. Thèse (Doctorat en sciences de l'éducation) — Université Paris, 2012.

ROIG, C. Symboles et société. Une introduction à la politique des symboles d'après l'œuvre de Kenneth Burke. Berne: Peter Lang, 1977.

WATZLAWICK, P. La réalité de la réalité. Paris: Seuil-Point, 2014.

ZAFFRAN, J.; VOLLET J. Zadig après l'école. Pourquoi les décrocheurs scolaires raccrochent-ils? Lormont: Le Bord de l'Eau, 2018.

Reçu : 02/10/2018

Received: 10/02/2018

Approuvé : 11/10/2018

Approved: 10/11/2018 